\title{
Medical Emergencies in Dental Practice
}

Authors: Orrett E. Ogle / Harry Dym / Robert J. Weinstock (Ed.)

Publisher: Quintessence Publishing

Language: English

ISBN: 978-0-86715-569-3

Edition: $1 / \mathrm{e}$

Publish Year: 2016

Pages: 200, illustrated

Price: $69.00 €$

Dental personnel must be trained to handle medical emergencies that occur during daily treatment. Yet, efficient management of medical emergencies should be thoroughly prepared in advance.

Drs Orrett E. Ogle, Harry Dym and Robert J. Weinstock's book entitled "Medical Emergencies in Dental Practice" develops a step-by-step treatment guide as well as decision-making algorithms for the immediate treatment of patients with medical emergencies.

In distinct and clearly structured chapters the readers are familiarized with pretreatment evaluation of the dental patient, essentials of an emergency kit and basic life support techniques.

Having acquired this knowledge, the readers are prepared to handle the most common medical emergency situations encountered during dental treatment such as: respiratory emergencies, acute chest pain, syncope, allergy and anaphylaxis, seizures, epilepsy, and stroke, nausea and vomiting, hemorrhagic emergencies, emergencies in the pregnant patient, hypertension and hypotension emergencies, TMJ emergencies, diabetic emergencies, malignant hyperthermia, thyroid crisis, local anesthesia emergencies, and adrenal crisis. This book provides an essential practical guide that should not be missing from the library of any dental clinic.

DOI: 10.25241/stomaeduj.2017.4(4).bookreview.6

Marian-Vladimir Constantinescu 